\title{
Exendin-4 improves glucose metabolism in insulin-resistant cells by upregulating the phosphorylative AMPK
}

\author{
Huaijie Wang ${ }^{1 \#}$, Han $\mathrm{Su}^{1,3 \#}$, Jian $\mathrm{Li}^{1,3}$, Shengyao $\mathrm{Ma}^{1}, \mathrm{Na} \mathrm{Li}^{1,4}$, Bin Jiang ${ }^{1}$, Meijia Zhang ${ }^{2}$, \\ Lianguang Huo ${ }^{1}$, Gong Dai ${ }^{1}$, Zhiqin Gao ${ }^{{ }^{*}}$, Xiaoyun Yang ${ }^{{ }^{*}}$, Meihua $\mathrm{Qu}^{12^{1}}$ *
}

${ }^{1}$ Key Laboratory of Applied Pharmacology, Weifang Medical University, Weifang, Shandong 261053, China; ${ }^{2}$ Weifang Second People's Hospital, Weifang Respectory Disease Hospital, Weifang, Shandong 261031, China; ' ${ }^{3}$ Department of Pharmacy, Affiliated Hospital of Weifang Medical University, Weifang, Shandong 261031, China; ${ }^{4}$ Medical Center, Ohio State University, Columbus, OH 43210, USA

${ }^{\#}$ These authors contributed equally to this study

${ }^{*}$ Corresponding authors: Meihua Qu, Weifang Medical University, 7166 Baotong West Street, Weifang, 261053 China. E-mail: qumeihua2016@ 163.com; Weifang Second People's Hospital, 7 Yuanxiao Street, Weifang, 261041 China; Xiaoyun Yang, Weifang Medical University, 7166 Baotong West Street, Weifang, 261053 China, E-mail: xiaoyuny1118@163.com; Zhiqin Gao, School of Bioscience and Technology, Weifang Medical University, 7166 Baotong West Street, Weifang, 261053 China. E-mail: zhiqingao2013@163.com

Received October 28, 2019; Accepted December 13, 2019

Glucagon-like peptide-1 (GLP-1) is a peptide with multiple functions in regulating blood glucose with the mechnism still not throughly understand. AMP-activated protein kinase (AMPK) plays an important role in glucose and energy homeostasis, especially in type 2 diabetes mellitus (T2DM) pathophysiology. This study explores GLP-1 analogue exendin-4 (Ex-4) regulating glucose balance by activating AMPK and regulation gluconeogenesis in insulin-resistant cell model (IR/ HepG2). IR/HepG2 cells were treated with Ex-4, AMPK activator or Ex-4 pretreated with AMPK inhibitor Compound C. The change of cell morphology, glucose consumption and lipid content in IR/HepG2 cells were examined. The expression of AMPK and p-AMPK in IR/HepG2 treated by Ex-4 were determined by Western blot and immunochemisthistology (IHC) assay. Key enzymes of glucose metabolism, phosphoenolpyruvate carboxykinase (PEPCK) and glucose-6-phosphatase (G-6-Pase) in IR/HepG2 cells treated by Ex-4 were detected by Western blot, immunohistochemistry and qRT-PCR. The results showed that Ex-4 treatment increased the expression of $p$-AMPK and reduced the expression of G-6-Pase and PEPCK in IR/HepG2 cells. Pretreatment with AMPK inhibitor Compound C counteracts the effect of Ex-4. These findings suggest that Ex-4 fulfilled it glucose regulation effect by phosphorylation of AMPK and decreased the expression of enzymes involving gluconeogenesis.

Key Words: Insulin resistance; IR/HepG2; GLP-1; Exendin-4; AMPK

\section{INTRODUCTION}

Type2 diabetes mellitus (T2DM) is commonly caused by the abnormal secretion of insulin or insulin resistance (IR). ${ }^{1,2}$ Insulin resistance is a process in which peripheral tissues, particularly the liver, muscle and fat, become resistant to insulin, which is a primary factor underlying the etiology of T2DM. $^{3}$ Therefore, developing antidiabetic drugs that exert effects on insulin sensitivity is critical for the cure of T2DM. AMP-activated protein kinase (AMPK) is a cellular nutrient sensor that affects cellular energy homeostasis., ${ }^{4,5}$ Once activated, AMPK plays a different role in cells by turning off energy-utilizing pathways and turning on energy-generating pathways. ${ }^{6}$ Studies have shown that AMPK increases insulin sensitivity in certain cells and improves metabolic milieu that may lead to reducing the risk of T2DM. ${ }^{7}$

Glucagon-like peptide-1(GLP-1) is an incretin secreted by L-cells

This is an open access journal, and articles are distributed under the terms of the Creative Commons Attribution-NonCommercial-ShareAlike 4.0 License, which allows others to remix, tweak, and build upon the work non-commercially, as long as appropriate credit is given and the new creations are licensed under the identical terms.

For reprints contact: weda-h@ weda-h.org

How to cite this article: Wang HJ, Su H, Li J, Ma S, Li N, Jiang B, Zhang M, Huo L, Dai G, Gao Z, Yang X, Qu M. Exendin-4 improves glucose metabolism in insulin-resistant cells by upregulating the phosphorylative AMPK. J ADV HEALTH 2019; 1(4): 234-238. 
of the small intestine, which promotes the $\beta$ cells of the pancreas to secrete insulin after food intake. ${ }^{4,8,9}$ GLP-1 analogue has become a second-line therapeutic drug for T2DM treatment. ${ }^{10,11}$ GLP-1 analogue, Exendin-4 (Ex-4), has been used in clinical treatment of type 2 diabetes mellitus (T2DM). ${ }^{12,13}$

Recent studies have found that GLP-1 promotes the phosphorylation of AMPK in hepatocytes and reduces the glucose content of hepatocytes in diabetic mice. ${ }^{13}$ Other studies showed that exenatide had direct protective effects on endothelial cells through the AMPK/Akt pathway in a GLP-1 receptor-dependent manner. ${ }^{14,15}$ However, the interaction of GLP-1 and AMPK in the treatment of diabetes is not clear. In this study, we explored the mechanism of GLP-1 analogue Exendin-4 (Ex-4) activated AMPK and decreased the key enzymes involving glucogenesis in insulin-resistant (IR) HepG2 cells.

\section{MATERIALS AND METHODS}

\section{Reagents}

Dulbecco's Modified Eagle's Medium (DMEM) supplements were purchased from Hyclone (UT, USA). Fetal bovine serum (FBS), bovine insulin was purchased from Solarbio Life Science (Beijing, China). Rabbit anti-AMPK and Rabbit anti-p-AMPK were obtained from Cell Signaling Technology (MA, USA). Rabbit anti-PEPCK and rabbit anti-glucose-6-phosphatase (G-6-Pase) were obtained from Abcam (MA, USA). Rabbit anti-GAPDH was obtained from Biosharp (Hefei, China). The IR Dye 800CW Goat anti-Rabbit antibody was from Gene Co (LI-COR, Nebraska, USA). The horse reddish peroxidase (HRP)-linked goat anti-rabbit antibody was obtained from DAKO (Beijing, China). BCA protein assay kit and TRIzol were purchased from CWBIO (Beijing, China). The qPCR RT kit and SYBR Green Real-time PCR Master Mix were obtained from Toyobo (Osaka, Japan). Ex-4, AMPK activator ARCAR and AMPK inhibitor Compound C (CC) were obtained from Med Chem Express (MCE, USA). The glucose oxidase-peroxidase (GOD-POD) kit was obtained from Applygen Technologies Inc (Beijing, China).

\section{Establishing the IR/HepG2 cell model}

HepG2 cells were obtained from the Chinese Academy of Sciences Cell Bank (Shanghai, China), and cultured in high glucose DMEM with $10 \% \mathrm{FBS}, 100 \mathrm{IU} / \mathrm{mL}$ penicillin and $100 \mathrm{mg} / \mathrm{mL}$ streptomycin in an incubator with humidified atmosphere and $5 \% \mathrm{CO}_{2}$ at $37^{\circ} \mathrm{C}$. HepG2 cells were seeded in 96 -well plate at $5 \times 10^{4}$ cells/well in high glucose DMEM with 2\% FBS and cultured for $24 \mathrm{~h}$ to reach $80 \%$ confluence. After $12 \mathrm{~h}$ of serum starvation, the cells were stimulated with various concentrations $\left(10^{-5}, 10^{-6}, 10^{-7}\right.$ or $\left.10^{-8} \mathrm{~mol} / \mathrm{L}\right)$ of insulin for $12,24,36$ or $48 \mathrm{~h}$. The glucose consumption in cell supernatant was measured using GOD-POD kit. It was found that IR/HepG2 cell model was established with the stimulation of insulin at $10^{-7}$ $\mathrm{mol} / \mathrm{L}$ for $36 \mathrm{~h}$.

\section{IR/HepG2 cell treatment}

IR/HepG2 cells were incubated in serum-free DMEM to reach 70\% confluence and then treated with Ex-4 (100nM), AICAR(1mM) for $24 \mathrm{~h}$ or preincubated with Compound $\mathrm{C}(40 \mu \mathrm{M})$ for $2 \mathrm{~h}$ before treated with Ex-4 for $24 \mathrm{~h}$. IR/HepG2 cells without treatment were used as untreated control.

\section{Determining the glucose consumption by oxidase-peroxidase Assay}

The supernatant of cells with different treatments was collected and glucose consumption of cells was determined by glucose GODPOD kit (Sigma Co., MO, USA) according to the manufacturer's instructions.

\section{Oil red O staining}

Oil red $\mathrm{O}$ staining was used to identify exogenous and endogenous lipid deposits. ${ }^{16} \mathrm{IR} / \mathrm{HepG} 2$ cells seeded in 6-well plates were fixed in $4 \%$ paraformaldehyde for $20 \mathrm{~min}$, treated by $60 \%$ isopropanol, and stained with a freshly prepared Oil Red O solution $\left(\mathrm{H}_{2} \mathrm{O}\right.$ : Oil Red O stock solution = 4:6; Oil Red O stock solution was $0.5 \%$ Oil Red O in isopropanol) for $10 \mathrm{~min}$. Cell nuclei were stained with hematoxylin. Images were obtained by a microscope (Nikon Eclipse Ti-SR, Tokyo, Japan).

\section{Immunocytochemical staining}

IR/HepG2 cells were grown on coverslips in a 6-well plate and treated with Ex-4 (100nM), AICAR (1mM) or Compound C as described above, fixed with $4 \%$ paraformaldehyde for $30 \mathrm{~min}$. After incubating in TBST containing $3 \%$ bovine serum albumin (BSA) for $1 \mathrm{~h}$ at room temperature, the cells were incubated overnight at $4{ }^{\circ} \mathrm{C}$ with a primary rabbit anti-p-AMPK (1:200), rabbit anti-PEPCK (1:100) and rabbit anti-G-6-Pase (1:100) diluted in TBST containing $5 \%$ skim milk. Cells were washed with TBST 3 times for $10 \mathrm{~min}$ each and incubated for $1 \mathrm{~h}$ with HRP-linked goat anti-rabbit IgG (1:500) at room temperature. Cells were washed with TBST 3 times for $10 \mathrm{~min}$ before diaminobenzidine (DAB) was added. The localization and expression of p-AMPK, G-6-Pase and PEPCK were observed under a Nikon Eclipse Ti-SR microscope (Tokyo, Japan).

\section{Quantitative real-time PCR}

Total RNA of HepG2 and IR/HepG2 cells was extracted by TRIzol reagent and dissolved in DEPC-treated distilled water. Total RNA $(1 \mu \mathrm{g})$ was reversely transcribed into cDNA using a Toyobo Rever Tra Ace qPCR-RT kit. The primers were designed by prism 5 software and the sequences were listed as follows $\left(5^{\prime}-3^{\prime}\right)$ : AMPK: F-CTCACCTCCTCCAAGTTATT and R-TCAGATGGGCTTATACAGC; PEPCK: F-TGCATGAAAGGTCGCACCA and R-CACAGAATGGAGGCATTT ; G-6-Pase: F-TGCTTTCTTCCACTCAGGCA and R-TGCTTTCTTCCACTCAGGCA; GAPDH: F-TCAACGACCACTTTGTCAAGCTCA and R-CTGGTGGTCCAGGGGTCCTTACT. qRT-PCR of these genes was performed using an SYBR Green fluorescent PCR kit. The PCR program was $95^{\circ} \mathrm{C}$ for $10 \mathrm{~min}$ and 40 cycles at $95^{\circ} \mathrm{C}$ for $5 \mathrm{~s}, 55^{\circ} \mathrm{C}$ for $10 \mathrm{~s}$, and $72{ }^{\circ} \mathrm{C}$ for $15 \mathrm{~s}$, following $7 \mathrm{~min}$ at $72^{\circ} \mathrm{C}$. All samples were analyzed using $2^{-\Delta \Delta_{\mathrm{Ct}}}$ method in parallel for GAPDH expression.

\section{Western blot analysis}

Drugs treated HepG2 and IR/HepG2 cells were harvested and lysed in lysis buffer. $40 \mu \mathrm{g}$ total protein was loaded in each well and run in $10 \%$ SDS-polyacrylamide gels at $110 \mathrm{~V}$ for $2 \mathrm{~h}$. The proteins were transferred to PVDF membrane at $300 \mathrm{~mA}$ for $100 \mathrm{~min}$ at $4^{\circ} \mathrm{C}$. After incubation in blocking solution ( $5 \%$ skim milk in TBST), membranes were incubated with antibodies overnight at $4^{\circ} \mathrm{C}$. The 
antibodies used were AMPK (1:1000), p-AMPK (1:1000), PEPCK (1:400), G-6-Pase (1:500) and GADPH (1:1000). Membranes were washed 3 times for $10 \mathrm{~min}$ by TBST, and incubated with IRDye $800 \mathrm{CW}$-conjugated goat anti-rabbit antibody (1:10000). Protein bands were visualized by an Odyssey Infrared Imaging System (LI-COR Biosciences, Lincoln, USA), and the intensity of each band was quantified using the Image J software.

\section{Statistical analysis}

The data were expressed as the mean \pm SD. SPSS software (SPSS Inc., Chicago, USA) was used for graphical representation and statistical analysis. One way ANOVA with Tukey's and S-N-K post hoc test was employed for multiple comparisons. Values of $P<0.05$ were considered statistically significant.

\section{RESULTS}

\section{Establishment of the insulin-resistant IR/HepG2 cell model}

Insulin resistant IR/HepG2 cell model was successfully established by treating HepG2 cells with high glucose and high concentration of insulin. The established insulin-resistant cells were evaluated by glucose consumption assay. ${ }^{17}$ The results showed that HepG2 cells treated with $10^{-7} \mathrm{~mol} / \mathrm{L}$ insulin for $36 \mathrm{~h}$ significantly reduced the glucose consumption (Table 1), indicating the successful establishment of insulin resistant cell model. The glucose consumption decreased in IR/HepG2 cells compared to HepG2 cells $(P<0.001)$ (Fig. 1A). The expression of G-6-Pase and PEPCK were used to evaluate glucose metabolism in IR/HepG2 cells. Western bolt results showed G-6-Pase and PEPCK expression significantly increased in IR/HepG2 cells comparing to control HepG2 cells (Fig. 1B). Figure $1 \mathrm{C}$ and figure $1 \mathrm{D}$ showed G-6-Pase and PEPCK expression in IR/HepG2 cells increased 1.5 and 2fold comparing to HepG2 cells calculated by Image J software, respectively. Oil red O staining was performed to observe the changing of fat metabolism in IR/HepG2 cells. The results showed increased fat droplets accumulated in IR/HepG2 cells compared to HepG2 cells (Fig. 1E).

\section{Ex-4 increased AMPK phosphorylation in IR/HepG2 cells}

To explore whether Ex-4 affected glucose metabolism by activating AMPK in insulin-resistant HepG2 cells, the expression of AMPK and phospho-AMPK were examined by Western blotting and IHC. AICAR is an activator of AMPK and is used as a positive control, while Compound $\mathrm{C}$ is commonly used as an inhibitor of AMPK and is used as a negative control in this study. Insulin resistant HepG2 cells were treated by Ex-4, AMPK activator AICAR, and Ex-4 pretreated by AMPK inhibitor Compound C, respectively. Western blot results showed Ex-4 and AMPK activator AICAR significantly increased the phosphorylation of AMPK, no significantly changing of AMPK expression was observed. Compound C pretreating attenuated increasing phosphorylation of AMPK effect by Ex-4 (Fig. 2A and Fig 2B). These data indicated that Ex-4 increased the protein phosphorylation of AMPK, but Ex-4 has no effect the protein phosphorylation of AMPK pretreated with Compound C. Immunocytochemistry showed similar expression trends in comparison of these four groups as Western blot (Figure 2C). It demonstrated that the role of GLP-1 in glucose metabolism is associated with AMPK. The protein of G-6-Pase and PEPCK is dramatically reduced after using Ex-4 and Ex-4 can improve glucose metabolism in IR/HepG2 cells. Our results demonstrated that Ex-4-AMPK signaling pathway is important in improving glucose metabolism.
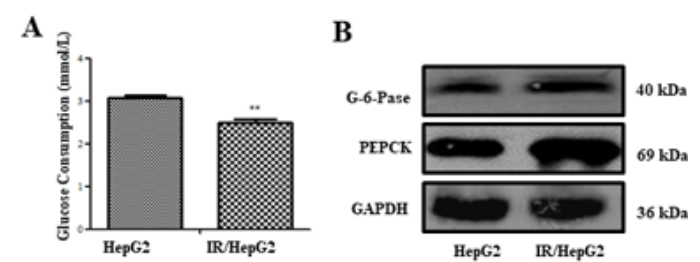

C

D
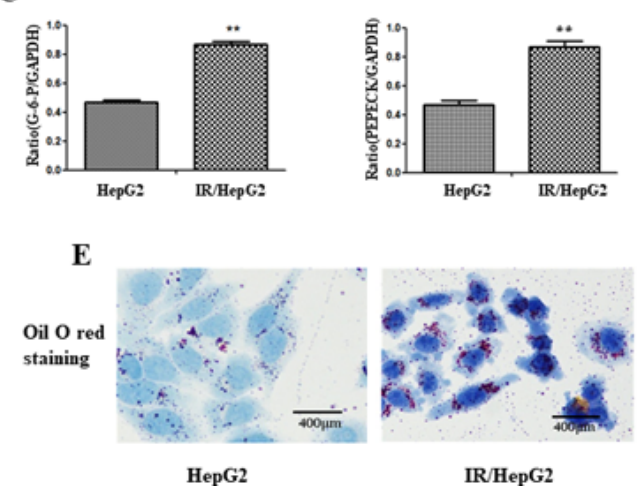

Figure1. The establishment of insulin-resistant IR/HepG2 cell model. A: The glucose consumption of HepG2 and IR/HepG2 cells. ${ }^{* *} P<0.01$, vs HepG2. B: Western blot on the expression of G-6-Pase and PEPCK in IR/HepG2 and HepG2 cells. C, D: Quantitative analysis of protein expression of G-6-Pase and PEPCK by image $\mathrm{J}$ software. ${ }^{* *} P<0.01$, vs HepG2. E: Oil red staining on IR/HepG2 and HepG2 cells. 40X.

Table 1. Glucose consumption with different concentrations of insulin (mM) in HepG2 cell supernatants

\begin{tabular}{cccccc}
\hline & \multicolumn{5}{c}{ Concentration of insulin $(\mathrm{mM})$} \\
\cline { 2 - 5 } & $10^{-5}$ & $10^{-6}$ & $10^{-7}$ & $10^{-8}$ & control \\
\hline $12 \mathrm{~h}$ & $3.02 \pm 0.1$ & $2.90 \pm 0.04^{*}$ & $2.73 \pm 0.09^{* *}$ & $2.98 \pm 0.04$ & $3.15 \pm 0.07$ \\
$24 \mathrm{~h}$ & $2.89 \pm 0.07$ & $2.81 \pm 0.03^{* *}$ & $2.65 \pm 0.04^{* * *}$ & $2.85 \pm 0.06^{* *}$ & $3.07 \pm 0.08$ \\
$36 \mathrm{~h}$ & $2.77 \pm 0.04^{* *}$ & $2.75 \pm 0.04^{* *}$ & $2.55 \pm 0.05^{* *}$ & $2.79 \pm 0.04^{* *}$ & $2.92 \pm 0.04$ \\
$48 \mathrm{~h}$ & $2.80 \pm 0.2$ & $2.76 \pm 0.7^{*}$ & $2.68 \pm 0.03^{* *}$ & $2.80 \pm 0.07$ & $2.91 \pm 0.04$ \\
\hline
\end{tabular}

${ }^{\star} P<0.05,{ }^{* *} P<0.01,{ }^{* *} P<0.001$ vs. Control. Data presented as Mean \pm SD.

Ex-4 improved glucose metabolism and decreased the expression of G-6-Pase and PEPCK in IR/HepG2 cells

The glucose consumption of IR/HepG2 cells treated by Ex-4, AMPK activator ARCAR, and Ex-4 pretreated by Compound C was evaluated using the GOD-POD assay kit. The results showed that glucose consumption significantly increased in Ex-4 and AICAR treated IR/HepG2 cells. Pretreating with Compound C attenuated the increased glucose consumption by Ex-4 (Fig. 3A).

In order to understand the molecular mechanism of Ex-4 in glucose metabolism regulation, G-6-Pase and PEPCK expression in different agents treated IR/HepG2 cells by qRT-PCR and Western blot. qRT-PCR data showed that Ex-4 and AICAR significantly reduced the mRNA expression of G-6-Pase and PEPCK in IR/HepG2 cells. Pretreated by compound C reversed Ex-4 function in decreasing G-6-Pase and PEPCK mRNA expression (Fig. $3 \mathrm{~B}-\mathrm{C})$. Figure $3 \mathrm{D}-\mathrm{F}$ showed the Western bolot results of the four 
groups. Figure $3 \mathrm{G}$ showed the IHC results also indicated that Ex-4 decreased the expression of G-6-Pase and PEPCK in IR/HepG2 cells. Blocking AMPK by Compound $\mathrm{C}$ reversed the effect of Ex-4 (Fig. 3G).

$\mathbf{A}$
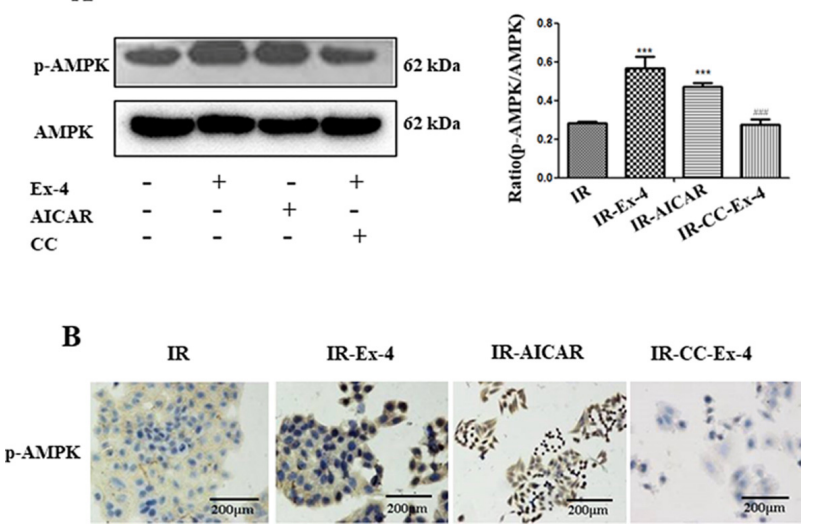

Figure2. Ex-4 increases phosphorylation of AMPK. A: Western blot on the expression of AMPK and p-AMPK in IR/HepG2 cells treated with Ex-4, AICAR or Ex-4 preincubated with CC. B: The relative p-AMPK/ AMPK ratios were calculated by Image $J$ software. Data are presented as mean \pm $\mathrm{SD}, n=3-5$ in each group. ${ }^{* *} P<0.01$, vs IR/hepG2 untreated; ${ }^{\# \#} P<0.01$ vs IR/HepG2 treated by Ex-4. C: p-AMPK in IR/HepG2 cells treated by different agents as described above was evaluated by Immunocytochemical assay.
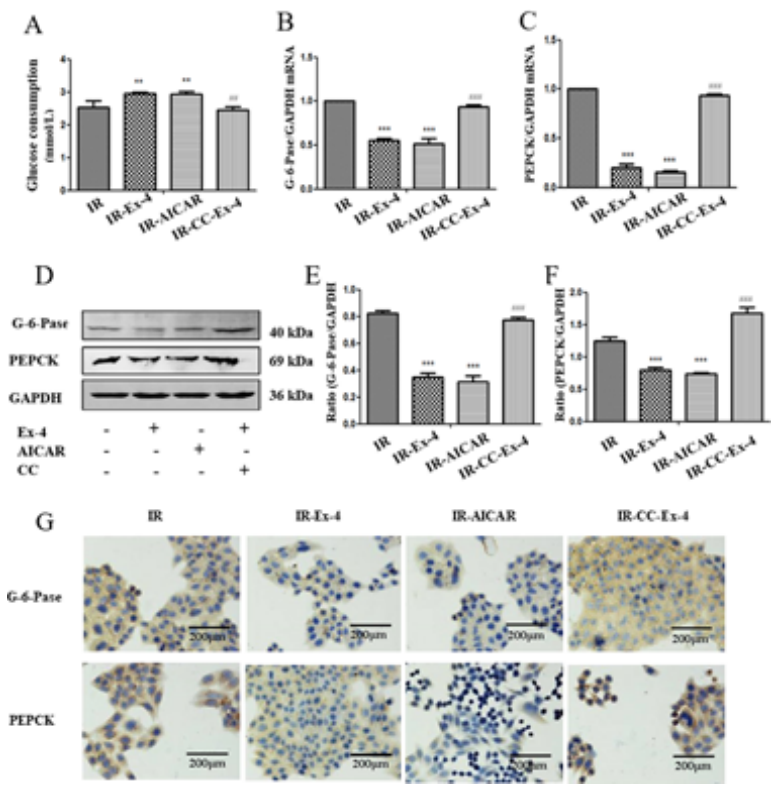

Figure3. Ex-4 improvs glucose metabolism in IR/HepG2 Cells. A: The glucose consumption of IR/HepG2 cells treated with Ex-4, AICAR, CC-Ex-4. B, C: The relative mRNA level of G-6-Pase and PEPCK in IR/HepG2 cells treated by different agents evaluated by quantitative real-time PCR, GAPDH served as internal reference control. D: G-6-Pase and PEPCK expression in IR/HepG2 cells treated by different agents detected by western blot. E, F: PEPCK and G-6-Pase expression of Fig 3D ananlysed by image J software. ${ }^{* *} \mathrm{P}<0.01$, IR-Ex-4 vs IR, IR-AICAR $v s$ IR; ${ }^{\#} P<0.01$ IR-CC-Ex-4 vscounteracts IR-Ex-4. G: PEPCK and G-6-Pase in IR/HepG2 cells treated by different agents as described above was evaluated by Immunocytochemical assay. ${ }^{* *} P<0.001$, IR-Ex-4 vs IR, IR-AICAR vs IR; ${ }^{\#} \mathrm{P}<0.001$ IR-CC-Ex-4 vs IR-Ex-4.

\section{DISCUSSION}

Insulin resistance is a physiologic state of a declined response to insulin, which is mainly triggered by longterm hyperglycaemia and the main reason of T2DM. ${ }^{18}$ Insulin resistance is characterized by the inhibition of glucose uptake in insulin-sensitive tissues, such as liver, muscle and adipose tissues. ${ }^{19,20}$ The liver plays an important role in regulating energy balance and maintaining glucose homeostasis, and it is also the key organ in T2DM pathophysiology. ${ }^{20}$ HepG2 cells originate from liver cells and retain many characteristics of hepatocytes. In this study, HepG2 cells were induced to insulin-resistance HepG2 cells (IR/HepG2) by high glucose and high insulin treatment. ${ }^{21,22}$ Hepatic gluconeogenesis is a crucial process regulating glucose metabolism. It has been reported that increased hepatic gluconeogenesis is mainly caused by overexpression of two key gluconeogenetic enzymes, G-6-Pase and PEPCK. ${ }^{23,24}$ PEPCK and G-6-Pase expression in IR/HepG2 cells treated by Ex-4 or other reagents were also evaluated in this study.

Glucagon-like peptide-1 (GLP-1) receptor agonists and DDPV inhibitors are new drugs in T2DM treatment. ${ }^{25}$ It is reported that GLP-1 receptor agonist, the novel antidiabetic drug (Ex-4), can improve lipid deposition in hepatocytes. ${ }^{26,27}$

Studies indicated that activation of the central nerve system GLP-1 receptor improves T2DM via glucose metabolism dependent inhibition of central nerve system AMPK. ${ }^{28}$ AMPK activation accelerate fatty acid oxidation and glucose uptake, suppresses triglycerides production in skeletal muscle, which is necessary for the body to maintain the balance of blood glucose. ${ }^{29}$ In this study, AMPK and p-AMPK were examined to understand whether Ex-4 improves glucose metabolism by increasing phosphorylation of AMPK. It was found that Ex-4 ameliorated the hepatic glucose metabolism by activating AMPK activity and decreasing the key enzymes involving gluconeogenesis.

Taken together, our results indicate that Ex- 4 activating AMPK is the main mechanism in improving insulin resistance. Targeting Ex-4/ AMPK signaling pathway, especially AMPK activating, could be promising in the treatment of T2DM.

\section{Acknowledgments}

This work was supported by the National Natural Science Foundation of China (81871892, 31671208, 81471048, 81500798, and 81503108), Shandong Province Natural Science Foundation (ZR2015HL128) and Technology Development Plan of Weifang (2018YX027).

\section{Author contributions}

Meihua Qu, Zhiqin Gao and Xiaoyun Yang designed the project; Meihua Qu, Zhiqin Gao, Huaijie Wang and Han Su write the paper; Huaijie Wang, Han Su, Jian Li, Shengyao Ma, Na Li, Bin Jiang, Meijia Zhang, Lianguang Huo, Gong Dai, Xiaoyun Yang, performed the experiment and data analysis.

\section{Conflicts of Interest}

The authors declare no potential conflicts of interest associated with this research. 


\section{REFERENCES}

1. Cho NH, Shaw JE, Karuranga S, Huang Y, da Rocha Fernandes JD, Ohlrogge AW, Malanda B. IDF Diabetes Atlas: Global estimates of diabetes prevalence for 2017 and projections for 2045. Diabetes research and clinical practice 2018; 138: 271-81.

2. Kharroubi AT, Darwish HM. Diabetes mellitus: The epidemic of the century. World Journal of Diabetes 2015; 6(6): 850.

3. Cao Y, Li X, Lu C, Zhan X. The relationship between vitronectin and hepatic insulin resistance in type 2 diabetes mellitus. Endocrine journal 2018; 65(7): 747-753.

4. Andersson LE, Shcherbina L, Al-Majdoub M, Vishnu N, Arroyo CB, Aste Carrara J, Wollheim CB, Fex M, Mulder H, Wierup N, Spégel P. Glutamine-Elicited Secretion of Glucagon-Like Peptide 1 (GLP-1) is Governed by an Activated Glutamate Dehydrogenase. Diabetes 2018; 67(3): 372.

5. Xie F, Zhu J, Hou B, Wang Y, Meng F, Ren Z, Ren S. Inhibition of $\mathrm{NF}-\kappa \mathrm{B}$ activation improves insulin resistance of L6 cells. Endocrine Journal 2017; 64(7): 685-93.

6. Kim B, Kim MS, Hyun CK. Syringin attenuates insulin resistance via adiponectin-mediated suppression of low-grade chronic inflammation and ER stress in high-fat diet-fed mice. Biochem Biophys Res Commun 2017; 488(1): 40-5.

7. Kahn BB, Alquier T, Carling D, Hardie DG. AMP-activated protein kinase: ancient energy gauge provides clues to modern understanding of metabolism. Cell Metabolism 2018; 1(1): 15-25.

8. Ichikawa M, Hirayama T, Fukushima M, Kitazawa I, Kojima K, Sakai T, Takatsu Y, Ohtaki T. Glycosaminoglycan Conjugation for Improving the Duration of Therapeutic Action of Glucagon-Like Peptide-1. ACS Omega 2018; 3(5): 5346-5354.

9. Lee J, Hong SW, Park SE, Rhee EJ, Park CY, Oh KW, Park SW, Lee WY. Exendin-4 regulates lipid metabolism and fibroblast growth factor 21 in hepatic steatosis. Metabolism Clinical \& Experimental 2014; 63(8): 1041-8.

10. Zhang L, Zhang L, Li L, Hölscher C. Neuroprotective effects of the novel GLP-1 long acting analogue semaglutide in the MPTP Parkinson's disease mouse model. Neuropeptides, 2018: S0143417918300684.

11. Imoto H, Shibata C, Ikezawa F, Kikuchi D, Someya S, Miura K, Naitoh T, Unno M. Effects of duodeno-jejunal bypass on glucose metabolism in obese rats with type 2 diabetes. Surgery today 2014; 44(2): 340 .

12. Abdelwahed OM, Tork OM, Din MM. GE, Rashed L, Zickri M. Effect of glucagon-like peptide-1 analogue; Exendin-4, on cognitive functions in type 2 diabetes mellitus; possible modulation of brain derived neurotrophic factor and brain Visfatin. Brain Research Bulletin 2018; 70(1): 178.

13. Xiao Y, Han J, Wang Q, Mao Y, Wei M, Jia W, Wei L. A Novel Interacting Protein SERP1 Regulates the N-linked Glycosylation and Function of GLP-1 Receptor in the Liver. Journal of Cellular Biochemistry 2017.

14. Wei R, Ma S, Wang C, Ke J, Yang J, Li W, Liu Y, Hou W, Feng X, Wang G, Hong T. Exenatide exerts direct protective effects on endothelial cells through the AMPK/Akt/eNOS pathway in a GLP-1 receptor-dependent manner. American Journal of Physiology Endocrinology And Metabolism 2016; 310(11): E947-E957.

15. Xu WW, Guan MP, Zheng ZJ, Gao F, Zeng YM, Qin Y, Xue YM. Exendin-4 alleviates high glucose-induced rat mesangial cell dysfunction through the AMPK pathway. Cellular physiology and biochemistry 2014; 33(2): 423-32.

16. Liu Y, Major AS, Zienkiewicz J, Gabriel CL, Veach RA, Moore DJ,
Collins RD, Hawiger J. Nuclear Transport Modulation Reduces Hypercholesterolemia, Atherosclerosis, and Fatty Liver. Journal of the American Heart Association Cardiovascular \& Cerebrovascular Disease 2013; 2(2): e000093.

17. Parker HE, Gribble FM, Reimann F. The role of gut endocrine cells in control of metabolism and appetite. Experimental Physiology 2014; 99(9): 1116-20.

18. Ji WW, Yu DA, Fan M, You M, Lu Y, Li EB, Xie N, Yan SS. Effects of GW002, a novel recombinant human glucagon-like peptide-1 (GLP-1) analog fusion protein, on CHO recombinant cells and BKS- $\mathrm{db}$ mice. Acta Diabetologica 2017; 54(11): 1-9.

19. Geng S, Wang S, Zhu W, Xie C, Li X, Wu J, Zhu J, Jiang Y, Yang X, Li Y, Chen Y, Wang X, Meng Y, Zhu M, Wu R, Huang C, Zhong C. Curcumin attenuates BPA-induced insulin resistance in HepG2 cells through suppression of JNK/p38 pathways. Toxicology Letters 2017; 272: 75.

20. Lee J, Hong SW, Park SE, Rhee EJ, Park CY, Oh KW, Park SW, Lee WY. Exendin-4 Inhibits the Expression of SEPP1 and Fetuin-A via Improvement of Palmitic Acid-Induced Endoplasmic Reticulum Stress by AMPK. Endocrinology \& Metabolism 2015; 30(2): 177-84.

21. Wang C, Li Y, Hao M, Li W. Astragaloside IV Inhibits Triglyceride Accumulation in Insulin-Resistant HepG2 Cells via AMPK-Induced SREBP-1c Phosphorylation. Frontiers in Pharmacology 2018; 9: 345.

22. Lee J, Yang J, Jeon J, Sang Jeong H, Lee J, Sung J. Hepatoprotective effect of esculetin on ethanol-induced liver injury in human HepG2 cells and C57BL/6J mice. Journal of Functional Foods 2018; 40: 536-543.

23. Andrés ML, Petra HO, Laura N, Francesc VA, Perales JC. Mitochondrial phosphoenolpyruvate carboxykinase (PEPCK-M) is a pro-survival, endoplasmic reticulum (ER) stress response gene involved in tumor cell adaptation to nutrient availability. Journal of Biological Chemistry 2014; 289(32): 22090-102.

24. Muzik O, Burghardt P, Yi Z, Kumar A, Seyoum B. Successful metformin treatment of insulin resistance is associated with down-regulation of the kynurenine pathway. Biochemical \& Biophysical Research Communications 2017; 488(1).

25. Tran S, Retnakaran R, Zinman B, Kramer CK. Efficacy of glucagon-like peptide-1 receptor agonists compared to dipeptidyl peptidase- 4 inhibitors for the management of type 2 diabetes: A meta-analysis of randomized clinical trials. Diabetes Obesity \& Metabolism 2018; 20(S1).

26. Xin Y, Zhang D, Fu Y, Wang C, Li Q, Tian C, Zhang S, Lyu X. C1qTNF-related protein 1 improve insulin resistance by reducing phosphorylation of serine 1101 in insulin receptor substrate. Endocrine Journal 2017; 64(8): 787.

27. Wang Y, Parlevliet ET, Geerling JJ, van der Tuin SJ, Zhang H, Bieghs V, Jawad AH, Shiri-Sverdlov R, Bot I, de Jager SC, Havekes LM, Romijn JA, Willems van Dijk K, Rensen PC. Exendin-4 decreases liver inflammation and atherosclerosis development simultaneously by reducing macrophage infiltration. Br J Pharmacology 2014; 171(3): 723-34.

28. Wu T, Zhang F, Yang Q, Zhang Y, Liu Q, Jiang W, Cao H, Li D, Xie S, Tong N, He J. Circulating mesencephalic astrocyte-derived neurotrophic factor is increased in newly diagnosed prediabetic and diabetic patients, and is associated with insulin resistance. Endocrine Journal 2017; 64(4): 403-10.

29. Yun YS, Noda S, Shigemori G, Kuriyama R, Takahashi S, Umemura M, Takahashi Y, Inoue H. Phenolic diterpenes from rosemary suppress cAMP responsiveness of gluconeogenic gene promoters. Phytotherapy Research 2013; 27(6): 906-10. 